\title{
BMJ Open HIV status and sexual behaviour among gay men in Ottawa: considerations for public health
}

\author{
Patrick O’Byrne, J Craig Phillips, Cynthia Kitson, Alyssa Bryan
}

To cite: O'Byrne $\mathrm{P}$

Phillips JC, Kitson C, et al. HIV status and sexual behaviour among gay men in Ottawa: considerations for public health. BMJ Open 2014:4:e005065

doi:10.1136/bmjopen-2014005065

- Prepublication history for this paper is available online. To view these files please visit the journal online (http://dx.doi.org/10.1136/ bmjopen-2014-005065).

Received 18 February 2014 Revised 19 June 2014 Accepted 1 August 2014
CrossMark

School of Nursing, University of Ottawa, Ottawa, Ontario, Canada

Correspondence to Dr Patrick O'Byrne; pjobyrne@uottawa.ca

\begin{abstract}
Objectives: HIV prevention efforts, particularly among men who have sex with men (MSM), have not achieved maximum effectiveness. A survey of MSM in Ottawa, Canada was completed to ascertain whether there were differences in how the perceived HIV status of participants and their partners influenced sexual practices.
\end{abstract}

Methods: Self-directed surveys were administered to a convenience sample of 721 MSM in Ottawa, Canada from November 2011 through May 2012. Data collection occurred at 14 sites. The survey identified whether participants identified as HIV positive, negative or unsure of their HIV status.

Results: The findings indicated variation between HIVnegative MSM and those who are unsure of their HIV status. Men who were unsure of their HIV status were less likely to report that they asked sexual partners or have had their partners ask about HIV status.

Conclusions: The results of this study indicate that some MSM may base decisions about HIV prevention on discussion about HIV status with their partners, rather than condom use. These practices may increase, rather than decrease, HIV transmission. Survey findings and extant literature demonstrate a need to inform MSM about the limitations of serosorting as a prevention strategy, and to provide facilitated access to sexually transmitted infection testing and treatment to further reduce onward HIV transmission.

\section{INTRODUCTION}

From public health and HIV prevention perspectives, the benefits of discussions about HIV status prior to sexual contact are debatable. ${ }^{1}$ In some research involving diagnosed HIV-positive persons, HIV-status disclosure corresponded with protected sex, whereas in other studies, there was either no change in sexual practices, or unprotected sex occurred after disclosure; these findings were consistent when sexual partners were of concordant and discordant HIV status. ${ }^{2-5}$ Similarly, among reportedly HIV-negative persons, researchers found that $\mathrm{HIV}$-status discussions were inconsistent or did not occur and, at

\section{Strengths and limitations of this study}

- Participants who were unsure of their HIV status were less likely to ask partners about HIV status, highlighting that it may be methodologically inappropriate to combine the data of persons of known versus unknown HIV status.

- Some gay men may use discussions about HIV status as a primary HIV prevention strategy. Owing to the proportion of persons unaware they are HIV positive, this prevention strategy could exacerbate transmission.

- HIV prevention workers should discuss serosorting, including a presentation of its merits and limitations. As part of this, access to sexually transmitted infection/HIV testing services would be recommended.

- More data are required about how gay men operationalise HIV prevention, specifically in relation to how they establish the HIV status of partners (whether overtly or otherwise).

- Researchers need to examine how criminal laws that enforce HIV-status disclosure are beginning to (if at all) effect serosorting and HIV prevention practices.

times, corresponded with unprotected sex when partners believed they had concordant HIV statuses. ${ }^{6-8}$ In other cases, HIV-negative participants reported that, in some instances, they engaged in unprotected sex with partners of discordant HIV status after disclosure, highlighting that the relationship between HIV-status disclosure and ensuing sexual practices is inconsistent. ${ }^{i 1}$

Nevertheless, due to the HIV prevention potential of behaviour change based on sex

${ }^{\mathrm{i}}$ Limiting discussions about HIV-status disclosure and ensuing sexual practices to whether or not unprotected sex occurs after disclosure is limited. After disclosure, for example, some persons may discover they have concordant HIV-statuses and engage in unprotected sex, while others may change their sex practices, for example, by engaging in oral rather than anal or vaginal sex. Available evidence does, however, highlight that unprotected sex does occur among persons with discordant HIV-statuses postdisclosure. 
partners' HIV status, this practice has been studied and named as serosorting; that is, "the practice of preferentially having sex with partners of concordant HIV-status or of selectively using condoms with HIV-discordant partners". ${ }^{9}$ Of interest, research has identified that, compared with nothing, this practice can prevent HIV transmission, although in limited ways. These shortcomings occur not only because a proportion of persons are unaware they are HIV positive (these numbers are detailed in the next section), but also because some persons appear to engage in 'seroguessing', in which they infer partners' HIV status using contextual clues rather than overt communication.

Because research has identified that this strategy is common among men who have sex with men (MSM) ${ }^{\text {ii }}$ to generate further data on the topic of HIV-status disclosure and HIV prevention among these men, we administered an exploratory survey to MSM in Ottawa, Canada. Taken as a whole, our findings suggested that first, in future research and prevention work, it might be appropriate to distinguish persons who report being HIV negative from those who indicate they are unsure of their HIV status and second, it may be necessary for researchers and prevention workers to reflect on presumptions that encouraging HIV-status discussions prevents HIV transmission. These findings challenge the premise that discussions about HIV status necessarily prevent forward transmission.

\section{THE LOCAL CONTEXT}

Epidemiological reports from the Public Health Agency of Canada ${ }^{10}$ indicated that in 2011, in Canada, there were approximately 3175 (range 2250-4100) incident infections. The largest burden $-46.6 \%$ ( $\mathrm{n}=1480$, range 1060-1900) - was among MSM, with an additional $2.5 \%(\mathrm{n}=80$, range 50-110) among MSM who also inject drugs. ${ }^{10}$ For prevalence, this same report estimated that as of 2011 there were approximately 71300 persons (range $58600-84000$ ) living with HIV in Canada. ${ }^{10}$ Nearly half of those living with HIV were MSM, i.e. $46.7 \%$ ( $\mathrm{n}=33$ 330, range 28 160-38 500) among MSM and $3.0 \%(\mathrm{n}=2160$, range 1520-2800) among MSM who inject drugs. ${ }^{10}$ The Public Health Agency of Canada ${ }^{10}$ report, lastly, contained estimates about the percentage of persons living with HIV who were undiagnosed and thus unaware of their HIV serological status. In 2011, this figure was estimated to be $20 \%$ of MSM, signalling that approximately 1 in $5 \mathrm{MSM}$ with HIV were unaware of their infection. ${ }^{10}$ As a final note, data from Ottawa conform to these distributions of HIV infection; MSM

\footnotetext{
${ }^{\mathrm{ii}}$ We use the term MSM to encompass gay, bisexual and all other men who have sex with men. While this term regrettably effaces sexual identity (including all social aspects related to a person's identification as gay or bisexual), we used it because our study focused on same-sex sexual behaviour between men, rather than perceptions of or ideas about sexual orientation and identity.
}

accounted for nearly half of both HIV incidence and prevalence figures. ${ }^{11}$

\section{SEROSORTING}

The extant literature on serosorting identifies that, because this practice should theoretically limit the possibilities for HIV transmission, it may be a sound prevention approach. ${ }^{12}$ That is, because HIV transmission requires that people with discordant HIV statuses engage in practices, sexual or otherwise, which transmit HIV, limiting such contacts ought to prevent transmission. However, an evaluation of 12449 clinical visits involving MSM in Seattle highlighted more HIV transmission among participants who reported serosorting, in comparison to those who reported condom use, but fewer HIV transmissions among serosorters when compared with participants reporting unprotected anal sex with serodiscordant partners. ${ }^{9}$ Similarly, Koblin et $a l^{13}$ identified, among 4295 MSM enrolled in an HIV prevention behavioural intervention in six US cities, that "just over one-fifth of seroincidence (21.6\%) was accounted for by unprotected receptive anal intercourse with partners believed to be HIV-negative" (p.735). Likewise, Buchbinder et $a l^{14}$ highlighted, in their prospective cohort study of 3257 MSM in nine US cities, that $28 \%$ of new infections in the study occurred among participants who reported only having HIV-negative sexual partners. Adding some clarification, Zablotska et als ${ }^{15}$ review of two observational cohorts in Australia identified that serosorting was sometimes based on presumptions about partners' HIV status, rather than discussions about HIV status. These authors ${ }^{15}$ referred to this practice as 'seroguessing.'

In real life, the benefits of serosorting may be limited because this practice relies on people knowing their HIV status, when there are persons (A) with diagnosed HIV infections, (B) with undiagnosed infections, (C) who erroneously believe they are HIV positive and (D) who correctly know they are HIV negative. ${ }^{9}{ }^{16-18}$ In the aforementioned HIV seroprevalence studies involving MSM, $41.2 \%, 44 \%$ and $31.1 \%$ of participants in the UK, the USA and Australia, respectively, were unaware of being HIV infected. ${ }^{16} 17{ }^{19}$ Another factor that could limit the HIV prevention effects of serosorting is that, as Truong $e t a l^{20}$ identified in their second-generation HIV surveillance programme in San Francisco, serosorting could correspond with increased transmission of other sexually transmitted infections (STIs), which could, in turn, exacerbate future HIV transmission. ${ }^{21} 22$

Consequently, serosorting, even when based on candid verbal discussion, likely results in some instances of people who are truly HIV negative engaging in unprotected receptive and/or penetrative anal sex with partners who have undiagnosed infections. ${ }^{9}{ }^{18}$ Because studies have linked the quantity of HIV virus with the probability of transmission, with higher viral levels corresponding with an increased potential for transmission, persons with undiagnosed HIV infection are likely more 
infectious than those with diagnosed infections. This occurs for two reasons. First, it is due to the natural progression of HIV-in that, during the acute infection period, persons can have upwards, and in excess, of one million HIV viral copies per millilitre of blood; second, once someone is diagnosed with HIV they can initiate HIV-antiretroviral treatment, which can effectively reduce their HIV viral load to undetectable levels (currently below 40 viral copies per millilitre of blood) ${ }^{23-26}$ Based on these data, researchers from the US Centers for Disease Control recently estimated that $54-70 \%$ of onward HIV transmission involves persons with undiagnosed HIV infections. ${ }^{27}$

\section{METHODOLOGICAL CONSIDERATIONS}

Before survey distribution began, the University of Ottawa Research Ethics Board approved this project. Thereafter, we piloted the survey with three distinct groups of MSM in Ottawa and made refinements as required. After these modifications, we distributed our surveys from November 2011 through May 2012 at a gay men's clinic for STI testing/treatment and HIV testing, two HIV treatment clinics, one bathhouse, two AIDS service organisations, four gay community events and four gay bars. To participate, persons had to identify as male, be at least 18 years old, be able to complete the survey in English or French and currently be or previously have been sexually active with men. Based on this process, we collected surveys from a convenience sample of 721 MSM who reside in Ottawa and its surrounding regions.

\section{Survey questions}

While an array of data were collected, for this paper we report: (A) basic demographic information, (B) sexual practices in the past 2 months and (C) self-reported perceptions about HIV-status discussions and willingness to engage in sexual activities with a partner with a discordant HIV status. The demographic component included questions about age, gender, income, ethnicity and education status.

The section about sexual practices inquired about participants' number of different sexual partners in the 2 months preceding survey completion, and whether the participant had, during these 2 months, engaged in oral, anal or vaginal sex, and if so, whether these sexual contacts were receptive and/or penetrative. Participants were asked whether condoms were used and if semen exchange occurred during these sexual contacts. If semen exchange had occurred, whether it was internal (mouth or anus) or external (face, chest, stomach, etc). The question about number of different sexual partners required participants to input a whole number, while the questions about semen exchange were written as, "I cum inside my partners':" or "My partners cum inside my:", or "I cum on my partners':" or "My partners cum on my:" with the options of "mouth", "vagina", "ass", "face", "chest", listed in the survey item. Participants were instructed to "Check all that apply". The option of "vagina" was included to ensure inclusivity of trans-men.

The final survey section about HIV-status discussions included questions about whether or not participants discussed their own or their sexual partners' HIV status and whether or not they would engage in sexual contact with a person with a discordant HIV status. These questions used a five-point scale (never, sometimes, $50 \%$ of the time, usually, always). For analysis, we coded this variable as 'never/sometimes' and 'usually/always'. In dichotomising the variable as such, it combined low and no levels of condom use and compared this behaviour to high levels of condom use, and consequently increased the number per group. The question about willingness to engage in sexual contact with a serodiscordant partner had the following response options: 'no', 'yes', 'unsure' and 'depends'; and it contained a blank line with 'depends on:', for participants to provide further details if they selected 'depends on'.

\section{Analysis}

We performed descriptive analyses ( $\chi^{2}$ tests) using SPSS V.19. Prior to analysis, we also established a $\mathrm{p}$ value of $<0.05$ as the measure of statistical significance.

\section{Procedures}

The steps in survey distribution were as follows. First, based on consultation with community partners and the pilot group participants, we compiled a list of gay venues in Ottawa. Second, we contacted each of these locations to request and obtain approval to distribute surveys on their premises. No site declined our request. Third, during on-site survey distribution, there were always at least two members of the research team present. One would circulate within the venue, soliciting participation, while the other would remain with the locked box where participants would deposit completed surveys. Both researchers, whether roaming or with the survey dropbox, however, inquired if potential participants would complete a short self-directed survey about their sexual practices, their STI/HIV testing practices and their perceptions about HIV. Potential participants were informed of the targeted research sample and assured anonymity. To maximise anonymity, participants were instructed to complete the survey on their own and to deposit the finished survey into a locked dropbox that would not be opened until the research team had left the premises. To facilitate survey completion, participants received a survey in their preferred language (French or English), a clipboard, a pen, a book light and a pair of reading glasses.

\section{RESULTS}

\section{Quantitative findings}

\section{Demographic information}

We obtained 721 completed surveys: $86.8 \%(\mathrm{n}=626)$ in English; $97.2 \% \quad(\mathrm{n}=701)$ by male participants; $81.8 \%$ $(n=590)$ by individuals who identified as Caucasian; $10 \%$ 
$(\mathrm{n}=72)$ who indicated they were HIV positive and $39.0 \%$ $(n=281)$ who reported a history of an STI diagnosis. Additionally, $81.9 \% \quad(n=590)$ of the sample reported college or university education, and $40.8 \%(\mathrm{n}=294)$ indicated a gross annual salary between $\$ 40000$ and $\$ 80000$ $(\mathrm{CAD})$. Lastly, the average age of the sample was 37 \pm 12 years.

\section{HIV-status discussions among respondents reporting HIV negative or unsure of HIV status}

Among all participants, 95.6\% $(\mathrm{n}=689)$ answered the question about HIV status: 72 (10.4\% of sample who reported their HIV status, and $10.0 \%$ of the total sample) were HIV positive, $69(9.6 \%)$ were unsure of their HIV status and 548 (79.4\% of sample who reported their HIV status and $76.0 \%$ of the total sample) were reportedly HIV negative. Of the HIV-negative participants, $34.8 \%(\mathrm{n}=187)$ reported they 'Never/Sometimes' ask sexual partners about their HIV status, while $60.9 \%$ $(\mathrm{n}=327)$ 'Usually/Always' ask. Among participants who were unsure of their HIV status, $50.0 \%(n=34)$ reported they 'Never/Sometimes' ask sexual partners about their HIV status and $40.9 \%(n=29)$ reported they 'Usually/ Always' ask. In comparison, $59.4 \% \quad(n=320)$ of HIV-negative participants reported their sex partners 'Never/Sometimes' ask about HIV status and 33.2\% $(n=179)$ reported their sexual partners 'Usually/Always' inquire. Among participants who were unsure of their HIV status, $71.0 \%(\mathrm{n}=49)$ reported their sexual partners 'Never/Sometimes' ask about HIV status and 27.3\% $(n=18)$ reported their sex partners 'Usually/Always' inquire. These differences were statistically significant, with more HIV-negative respondents reporting they 'Usually/Always' ask partners about their HIV status and more respondents who were unsure of their HIV status reporting they 'Never/Sometimes' ask about partners' HIV status $\left(\chi^{2}=7.35(1) \mathrm{p}=0.007\right)$. Therefore, we analysed persons who reported they were HIV negative and those who were unsure of their HIV status as distinct groups.

In addition, we observed significant relationships between unprotected receptive anal sex with internal ejaculation and HIV-status discussions. HIV-negative participants who reported that their partners ejaculate inside them without protection during anal sex were more likely to report that they 'Usually/Always' ask about HIV status, while those who did not let partners ejaculate inside them without condoms during anal sex were more likely to 'Never/Sometimes' inquire about HIV status $\left(\chi^{2}=14.97, \mathrm{p}<0.001\right.$; see table 1$)$. Similarly, HIV-negative participants who reported that their partners ejaculate inside them without protection during anal sex were more likely to report their partners 'Usually/Always' ask about HIV status, while those who did not allow partners to ejaculate inside them without protection during anal sex were more likely to report that their partners 'Never/Sometimes' ask about HIV status $\left(\chi^{2}=10.74, \mathrm{p}=0.001\right)$. Furthermore, HIV-negative participants who noted they ejaculate inside their
Table 1 HIV-status inquiries and sexual practices among HIV-negative respondents

\begin{tabular}{llrl}
\hline & \multicolumn{2}{l}{$\begin{array}{l}\text { Unprotected } \\
\text { receptive anal } \\
\text { sex with } \\
\text { internal } \\
\text { ejaculation }\end{array}$} & \\
\cline { 2 - 3 } Ask about HIV status & No & Yes & Total \\
\hline Never/sometimes & 109 & 21 & 130 \\
Usually/always & 161 & 87 & 248 \\
Total & 270 & 108 & 422 \\
\hline$\chi^{2}=14.97(1), p<0.001$. & & & \\
\end{tabular}

partners without protection during anal sex were more likely to report they 'Usually/Always' ask partners about HIV status, while those who reported they did not ejaculate inside their partners without protection during anal sex were more likely to report that they 'Never/ Sometimes' inquire about their partners' HIV status $\left(\chi^{2}=9.48, \quad \mathrm{p}=0.002\right)$. No significant findings were observed within any of the aforementioned categories for respondents who were unsure of their HIV status.

Lastly, HIV-negative participants who reported they would have sex with a person with HIV were more likely to 'Never/Sometimes' inquire about partners' HIV status, while those who were not willing to engage in sexual contact with someone with HIV were more likely to 'Usually/Always' ask about HIV status $\left(\chi^{2}=12.39\right.$, $\mathrm{p}<0.001$; see table 2). Again, no significant findings were observed for respondents reporting they were unsure of their HIV status.

\section{DISCUSSION}

In summary, in this research we observed differences between persons who identified as HIV negative and those who were unsure of their HIV status. Ostensibly HIV-negative persons were more likely to 'usually/ always' ask partners about their HIV status, while persons who reported being unsure of their HIV status were more likely to 'never/sometimes' ask. Moreover, among HIV-negative respondents, 'usually/always' discussing HIV status with partners was associated with

Table 2 HIV-status inquiries and willingness to have sex with persons with HIV among HIV-negative respondents

\begin{tabular}{lrrr}
\hline & \multicolumn{3}{l}{$\begin{array}{l}\text { Willing to } \\
\text { have sex with } \\
\text { person with } \\
\text { HIV }\end{array}$} \\
\cline { 2 - 4 } Ask about HIV status & No & Yes & Total \\
\hline Never/sometimes & 56 & 63 & 139 \\
Usually/always & 148 & 74 & 239 \\
Total & 228 & 150 & 378 \\
\hline$\chi^{2}=12.39(1), p<0.001$. & & & \\
\end{tabular}


receptive unprotected anal sex with internal ejaculation, while 'never/sometimes' discussing HIV status was associated with protected receptive anal sex. Lastly, HIV-negative respondents who reported they 'usually/ always' ask partners about their HIV status were less willing to have sex with persons with HIV; however, we observed no significant differences regarding the willingness of respondents who reported being unsure of their HIV status to engage in sexual contact with persons living with HIV.

First, these results indicate that researchers and HIV prevention works should consider persons who identify as HIV negative and those who are unsure of their HIV status as members of distinct behavioural groups. In this study, this distinction emerged regarding (A) an increased likelihood to ask sexual partners about their HIV status among HIV-negative respondents, when compared with persons who were unsure of their HIV status and (B) an elevated willingness of persons unsure of their HIV status to engage in sexual contact with persons living with HIV, when compared with persons who reported being HIV negative. Researchers have previously identified similar behavioural distinctions between persons who report being HIV negative and those who are unsure of their HIV status. Among persons HIV diagnosed, reportedly HIV negative and unaware of their HIV status, researchers have noted the lowest levels of HIV-status disclosure to persons of unknown HIV status, compared with persons who are either HIV negative or HIV positive. ${ }^{28} 29$

These results thus suggest that, for research and prevention work, the dichotomous categorisation of people's HIV status as either negative or positive may miss important nuances. Specifically, this classification does not account for persons with undiagnosed HIV infection, persons who are unaware of their HIV status and persons who erroneously believe they are HIV positive based on prior sexual behaviour. HIV seroprevalence studies involving MSM in the UK, the USA and Australia have demonstrated that these groups exist, ${ }^{16} 1719$ with members of each group being more likely to engage in practices that transmit HIV. ${ }^{16} 17$

Accordingly, we suggest that researchers and prevention workers should consider if it is appropriate to collapse people into the dichotomous groupings of HIV positive and HIV negative or, rather, if it would be more fitting to adopt a system which acknowledges that some persons have undiagnosed infections, others are unsure of their HIV status and some (who were not discussed in this research) incorrectly assume they are HIV positive. As observed in our study and previous research as well, ${ }^{16}{ }^{17}{ }^{19}$ differences in the sexual behaviours of members of these groups lead us to hypothesise that modified interventions and data collection tools may be better suited to ensure that the uniqueness of each group is accurately identified and adequately addressed. In light of ongoing HIV transmission among MSM, notwithstanding high levels of HIV medication usage by these men, perhaps more finely targeted interventions that are tailored to the unique cultural aspects and characteristics of these men and their communities are required.

Second, our results could suggest that some HIV-negative respondents may have either been serosorting (ie, attempting to exclusively engage in sexual contacts that could transmit HIV with persons who have similar HIV statuses), or at least they reported thought processes that resemble serosorting. ${ }^{18}$ Suggesting that serosorting may have been occurring-or at least that the participants consider HIV-status disclosure as a viable prevention strategy-are our findings that respondents who stated they were HIV negative were (A) more likely to ask partners about their HIV status and (B) less willing to have sex with persons living with HIV. Such findings have been identified previously when participants reported that they base decisions about condom use on their partners' reported HIV status. ${ }^{12} 132030$

A limitation of our data on the topic of serosorting, however, was that we did not directly ask respondents if they had ever actually changed their sexual practices when a person disclosed an HIV-positive status. Instead, we inferred that this practice may have been occurring based on our analysis of our HIV-negative respondents' reported behaviour (both sexual and related to HIV-status discussions), sexual behaviour changes and willingness to have sex with partners with discordant HIV statuses. Explicit questions on this topic could have provided more direct evidence on it, and thus should be garnered in future studies. Another limitation of our study is that it was based on a convenience sample of MSM who attended venues and events that gay men may attend (eg, bars, social events, STI testing clinics and bathhouses). Our results must therefore be interpreted in light of the fact that we sampled respondents from milieux targeted at gay men, which might have precluded the inclusion of MSM who do not identify as gay or bisexual, who avoid gay locales and events, who are not open about their sexual practices with men, and who use online services to organise their social and sexual lives. Additionally, these venues and events may not be frequented by all gay men and therefore may not be representative of all gay men living in Ottawa.

Third, these results maintain that the relationships between HIV-status disclosure, HIV prevention and public health are not clear-cut. While discussing HIV status may prevent transmission for some individuals, in other cases, relying on such disclosure can exacerbate HIV transmission because of persons who are unaware they are HIV positive and potentially able to transmit the virus to others. In these latter cases, persons who choose to engage in unprotected sex or who do not avoid practices that are known to more easily transmit HIV because they have inaccurate or inadequate information highlights the major limitations of promoting HIV-status disclosure as an efficacious HIV prevention approach. Accordingly, we believe that HIV prevention 
workers, and public health professionals specifically, should not categorically rely on or attempt to enforce HIV-status disclosure as a means of HIV prevention. Other options, such as condom use or the elimination of practices that most easily transmit HIV, would be preferable and have been documented as successful at preventing HIV transmission. Bearing this in mind, this does not mean that HIV disclosure discussions are without purpose, but rather, that they should not be overly emphasised as effective and efficacious prevention strategies. This is an important reflection for HIV prevention professionals as well as for persons affected by HIV when addressing the challenging topic of HIV prevention.

\section{FINAL REMARKS}

Of interest, our results identified noteworthy differences in sexual behaviour: first, between persons who reported being either unsure of their HIV status or HIV negative; and second, between persons who ask and do not ask partners about their HIV status. In combination with extant understandings about HIV transmission, these data signal that discussions about HIV status and serosorting likely hold some potential as efficacious HIV prevention strategies, although with many caveats that can limit effectiveness. Indeed, a number of items, detailed above, highlight that, instead of preventing HIV transmission in some instances, HIV-status discussions and serosorting can correspond with unintended exposures to HIV when sexual partners erroneously believe there is no possibility of transmission. Based on HIV seroprevalence studies from the UK, the USA and Australia, ${ }^{16} 1719$ because some persons believe they are HIV negative when they are not, unintended transmission could occur when HIV-negative persons engage in sexual contact with partners who inaccurately, but unintentionally, report they are HIV negative. In such situations, HIV-status disclosure can exacerbate, rather than ameliorate, persons' HIV prevention efforts.

For HIV prevention workers, we do not take this to mean that serosorting should be rejected as a viable HIV prevention strategy. Instead, we simply posit that prevention professionals should inform their clients and target populations about the current literature on and understandings of serosorting, specifying what it yields and how and when it appears to work. If a client's goal is to ensure that HIV transmission cannot occur, then they need to be informed that relying on discussions about HIV status, and not condoms, could result in them unknowingly coming into contact with HIV. However, if the client's intention is to mitigate, rather than eliminate, the possibility of HIV acquisition during unprotected sexual contact, then, as noted by Golden $e t a l,{ }^{9}$ serosorting is one method to reduce the chances of becoming HIV positive. To help ensure that serosorting offers such protection, we recommend that public health departments and authorities provide persons who engage in serosorting with facilitated access to HIV testing and STI testing/treatment to enhance HIV-status awareness and to minimise the contributory effects STIs have on HIV transmission. In the absence of such services, serosorting could induce a delayed increase in HIV transmission.

Lastly, the inconsistent relationships between HIV-status disclosure and ensuing sexual practices-an association that our data corroborate-highlights the limitations of promoting HIV-status disclosure as a prevention strategy, which leads us to question: if HIV-status disclosure can really only be interpreted as consistently valid when a person reports being HIV positive, in which case behaviour change and/or condoms are recommended, then why promote this strategy? Why not endorse other strategies? Further research is required to add more details on this topic and to help clarify, does promoting HIV-status disclosure actually affect HIV prevention, or is it simply an enforcement of social norms and legal recommendations?

Contributors PO'B conceived the study design and obtained funding. PO'B and $A B$ were involved in data collection. $P O{ }^{\prime} B, J C P, A B$ and $C K$ were involved in data analysis and synthesis, and manuscript preparation. All authors contributed substantially to this manuscript and approved the final version before submission

Funding This work was supported by an Early Researcher Award (PO'B) from the Government of Ontario Ministry of Research and Innovation.

Competing interests None.

Ethics approval University of Ottawa Research Ethics Board

Provenance and peer review Not commissioned; externally peer reviewed.

Data sharing statement No additional data are available.

Open Access This is an Open Access article distributed in accordance with the Creative Commons Attribution Non Commercial (CC BY-NC 3.0) license, which permits others to distribute, remix, adapt, build upon this work noncommercially, and license their derivative works on different terms, provided the original work is properly cited and the use is non-commercial. See: http:// creativecommons.org/licenses/by-nc/3.0/

\section{REFERENCES}

1. Simoni JM, Pantalone DW. Secrets and safety in the age of AIDS: does HIV disclosure lead to safer sex? Top HIV Med 2004;14:109-18.

2. Crepaz N, Marks G. Serostatus disclosure, sexual communication and safer sex in HIV-positive men. AIDS Care 2003;15:379-87.

3. Hightow-Weidman LB, Phillips G, Outlaw AY, et al. Patterns of HIV disclosure and condom use among HIV-infected young racial/ethnic minority men who have sex with men. AIDS Behav 2013;17:360-8.

4. Parsons JT, Schrimshaw EW, Bimbi DS, et al. Consistent, inconsistent, and non-disclosure to casual sexual partners among HIV-seropositive gay and bisexual men. AIDS 2005;19(Suppl 1): S87-97.

5. Raj A, Cheng DM, Levison R, et al. Sex trade, sexual risk, and nondisclosure of HIV serostatus: findings from HIV-infected persons with a history of alcohol problems. AIDS Behav 2006;10:149-57.

6. Dempsey AG, MacDonell KE, Naar-King S, et al. Patters of disclosure among youth who are HIV-positive: a multisite study. J Adoles Health 2012;50:315-17.

7. Klitzman R, Exner T, Correale J, et al. It's not just what you say: relationships of HIV disclosure and risk reduction among MSM in the post-HAART era. AIDS Care 2007;19:749-56.

8. Winter AK, Sullivan PS, Khosrpour CM, et al. Discussion of HIV status by serostatus and partnership sexual risk among internet-using MSM in the United States. J Acquir Immune Defic Syndr 2012;60:525-9. 
9. Golden MR, Stekler J, Hughes JP, et al. HIV serosorting in men who have sex with men: is it safe? J Acquir Immune Defic Syndr 2008;49:212-18.

10. Public Health Agency of Canada. [PHAC]. Summary: Estimates of HIV Prevalence and Incidence in Canada, 2011. 2012. http://webqa. phac-aspc.gc.ca/aids-sida/publication/survreport/estimat2011-eng.php

11. Ottawa Public Health. Sexually Transmitted Infections and Sexual Health in Ottawa, 2011. Health Status Report. http://ottawa.ca/ calendar/ottawa/citycouncil/obh/2011/11-21/F\%205794\%20OPH\% 20Sexual\%20Health\%20Report\%20Eng\%20WEB\%20Tagged\% 20Nov14-11.pdf

12. Mao L, Crawford J, van de Ven $\mathrm{P}$, et al. Differences between men who report frequent, occasional or no unprotected anal intercourse with casual partners among a cohort of HIV-seronegative gay men in Sydney, Australia. AIDS Care 2006b;18:942-51.

13. Koblin BA, Husnik MJ, Colfax G, et al. Risk factors for HIV infection among men who have sex with men. AIDS 2006;20:731-9.

14. Buchbinder SP, Vittinghoff $E$, Heagerty $P J$, et al. Sexual risk, nitrate inhalant use, and lack of circumcision associated with HIV seroconversion in men who have sex with men in the United States. $J$ Acquir Immune Defic Syndr 2005;39:82-9.

15. Zablotska IB, Imrie J, Prestage G, et al. Gay men's current practice of HIV serocondordtant unprotected anal intercourse: serosorting or seroguessing? AIDS Care 2009;21:501-10.

16. Pedrana AE, Hellard ME, Guy $\mathrm{R}$, et al. The difference in self-reported and biological measured HIV prevalence: implications for HIV prevention. AIDS Behav 2012;16:1454-63.

17. Williamson LM, Dodds JP, Mercey DE, et al. Sexual risk behaviour and knowledge of HIV status among community samples of gay men in the UK. AIDS 2008;22:1063-70.

18. Fernandez-Davila $\mathrm{P}$, Folch $\mathrm{C}$, Zaragoza Lorca K, et al. Silence and assumptions: narratives on the disclosure of HIV status to casual sexual partners and serosorting in a group of gay men in Barcelona. Int J Sex Health 2011;23:139-55.

19. Centers of Disease Control and Prevention (CDC). Prevalence and awareness of HIV infection among men who have sex with men-21 cities, United States, 2008. MMWR Morb Mortal Wkly Rep 2010;59:1201-7.
20. Truong HHM, Kellogg R, Klausner JD, et al. Increases in sexually transmitted infections and sexual risk behaviour without a concurrent increase in HIV incidence among men who have sex with men in

San Francisco: a Suggestion of HIV serosorting? Sex Transm Infect 2006;82:461-6.

21. Cohen MS, Hoffman IF, Royce RA, et al. Reduction of concentration of HIV-1 in semen after treatment of urethritis: implications for prevention of sexual transmission of HIV-1. Lancet 1997;349:1868-73.

22. Freeman EE, Weiss HA, Glynn JR, et al. Herpes simplex virus virus 2 infection increases HIV acquisition in men and women: systematic review and meta-analysis of longitudinal studies. AIDS 2006;20:73-83

23. Brenner BG, Roger M, Routy JP, et al. High rates of forward transmission events after acute/early HIV-1 infection. J Infect Dis 2007;195:951-9.

24. Chakraborty $\mathrm{H}$, Sen PK, Welms RW, et al. Viral burden in genital secretions determines male-to-female sexual transmission of HIV-1: a probabilistic empiric model. AIDS 2001;15:621-7.

25. Cohen MS, Chen YQ, McCauley M, et al. Prevention of HIV-1 infection with early antiretroviral therapy. $N$ Engl J Med 2011;365:493-505.

26. Quinn TC, Wawer MJ, Sewankambo N, et al. Viral load and heterosexual transmission of human immunodeficiency virus type I. N Engl J Med 2000;342:921-9.

27. Marks N, Crepaz N, Janssen RS. Estimating sexual transmission of HIV from persons aware and unaware that they are infected with the virus in the USA. AIDS 2006;20:1447-50.

28. Rouwenhorst E, Mallitt K-A, Prestage G. Gay men's use of condoms with casual partners depends on the extent of their prior acquaintance. AIDS Behav 2012;16:1589-96.

29. Semple SJ, Zians J, Grant I, et al. Sexual risk behaviour of HIV-positive methamphetamine-using men who have sex with men: the role of partner serostatus and partner type. Arch Sex Behav 2006;35:461-71.

30. Mao L, Crawford JM, Hospers HJ, et al. "Serosorting" in casual anal sex of HIV-negative gay men is noteworthy and is increasing in Sydney, Australia. AIDS 2006a;20:1204-6. 